\title{
SOME FAMILIES OF ISOPARAMETRIC HYPERSURFACES AND RIGIDITY IN A COMPLEX HYPERBOLIC SPACE
}

\author{
MICHEAL H. VERNON
}

\begin{abstract}
The geometric notion of equivalence for submanifolds in a chosen ambient space is that of congruence. In this study, a certain type of isoparametric hypersurface of a complex hyperbolic space form is shown to have a rigid immersion by utilizing the congruences of a Lorentzian hyperbolic space form that lies as an $S^{1}$-fiber bundle over the complex hyperbolic space. Several families of isoparametric hypersurfaces (namely tubes and horospheres) are constructed whose immersions are rigid.
\end{abstract}

\section{INTRODUCTION}

Analogous to the Hopf fibration $S^{1} \rightarrow S^{2 m+1} \rightarrow \mathrm{C} P^{m}$ of an odd-dimensional sphere over complex projective space, we have a Lorentzian $S^{1}$-fiber bundle, called anti-De Sitter spacetime (denoted by $H_{1}^{2 n+1}$ ), that lies over a complex hyperbolic space form $\mathrm{CH}^{n}(-4)$. The rigidity of an immersion of a hypersurface into $\mathbf{C} P^{m}$ is usually obtained by considering the $S^{1}$-fiber bundle over the hypersurface as a hypersurface of $S^{2 m+1}$ and then applying isometries of $\mathbf{R}^{2 m+1}$ that restrict to isometries of $S^{2 m+1}$. We shall use the same approach to establish the rigidity of certain isoparametrical real hypersurfaces of $\mathrm{CH}^{n}(-4)$.

Let $M^{2 n-1}$ be a complete, connected real hypersurface of $\mathrm{CH}^{n}(-4)$ with immersion $i$ and $\pi: H_{1}^{2 n+1} \rightarrow \mathbf{C} H^{n}$ be the Riemannian submersion with timelike, totally geodesic fibers. If $\widetilde{M}_{1}^{2 n}=\pi^{-1}(M)$ is the $S^{1}$-fiber bundle over $M$, there is an induced immersion $\tilde{i}: \widetilde{M} \rightarrow H_{1}^{2 n+1}$ such that the following diagram commutes:

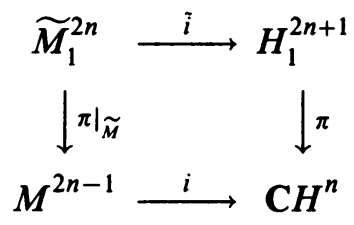

Received by the editors January 28, 1986 and, in revised form, October 29, 1987. Presented at the January 1986 meeting of the American Mathematical Society.

1980 Mathematics Subject Classification (1985 Revision). Primary 53C55; Secondary 53C50.

Key words and phrases. Real hypersurfaces, complex hyperbolic space, Lorentzian manifold, Anti-De Sitter spacetime, $S^{1}$-fibration, isoparametric hypersurfaces, tubes, rigidity. 
Now by considering the natural imbedding of $H_{1}^{2 n+1}$ into the complex Lorentzian euclidean space $\mathrm{C}_{1}^{n+1}$, we can view $\widetilde{M}$ as a Lorentzian submanifold of $\mathbf{R}_{2}^{2 n+2}$, of real codimension 2, and thereby use known congruence results for submanifolds of semi-Riemannian euclidean spaces to obtain a congruence result for hypersurfaces of $\mathrm{CH}^{n}$ :

Theorem 2. Let $M$ and $N$ be isoparametric orientable real hypersurfaces of $\mathrm{CH}^{n}$ that each have the distinguished direction $U$ as a principal direction. If $M$ and $N$ have the same second fundamental form, i.e., the corresponding principal curvatures are the same, then $M$ and $N$ are congruent.

This theorem establishes the rigidity of several families of isoparametric hypersurfaces of $\mathrm{CH}^{n}$ that are constructed in this study. These families form the model spaces for classifications of isoparametric hypersurfaces that will appear in other papers. Along the way, we will construct a horosphere and explicitly display the convergence of a family of geodesic hyperspheres to a horosphere using Theorem 2. The notion of a certain distinguished direction on a real hypersurface being a principal direction is central to the study.

This paper includes a portion of my doctoral dissertation at Michigan State University. I would like to extend my deepest appreciation to Gerald Ludden, Bang Yen Chen, David Blair, and Ralph Howard for sharing their knowledge and many valuable suggestions.

\section{REAL HYPERSURFACES OF $\mathrm{CH}^{n}(-4)$}

Let $\mathrm{CH}^{n}(-4), n \geq 2$, denote a complex hyperbolic space equipped with the Bergman metric tensor, i.e., a complex space form of constant holomorphic sectional curvature -4 . Let $M^{2 n-1}$ be an orientable real hypersurface of $\mathrm{CH}^{n}$, $\nabla$ and $\bar{\nabla}$ be the metric connections on $M$ and $C H^{n}$, respectively, so that the Gauss and Weingarten formulae can be written as

(1.1) $\bar{\nabla}_{X} Y=\nabla_{X} Y+\langle H X, Y\rangle \xi$ and $\bar{\nabla}_{X} \xi=-H X$ for all $X, Y \in T(M)$,

where $\xi$ is a unit normal field on $M$ in $\mathrm{CH}^{n}$ and $H$ denotes the second fundamental form (in this case the Weingarten map of $\xi$ in $\operatorname{End}[T(M)]$ ). We shall refer to the eigenvalues and eigenvectors of $H$ in $\mathbf{R}$ and $T(M)$, respectively, as principal curvatures and principal directions.

Let $J$ denote the complex structure of the ambient complex space form. It induces a bundle endomorphism $\phi$ of rank $2 n-2$ and a linear functional $f$ on $T(M)$ given by setting at each point $p$ of $M$

$$
J X=\phi X+f(X) \xi \text { for all } X \text { in } T_{p}(M) .
$$

Let $U=-J \xi$. As $M$ is of codimension one we have $U \in T(M)$. The following equations now hold for all $X, Y$ in $T(M)$ :

$$
\begin{gathered}
f(X)=\langle X, U\rangle, \\
f(\phi X)=0,
\end{gathered}
$$




$$
\begin{gathered}
\phi U=0, \\
\phi^{2} X=-X+f(X) U, \\
\langle X, \phi Y\rangle=-\langle\phi X, Y\rangle \\
\langle\phi X, \phi Y\rangle=\langle X, Y\rangle-f(X) f(Y) .
\end{gathered}
$$

$(\phi, f, U)$ is an example of what is called an almost contact structure on $M$. The tensor fields $\phi$ and $U$ have the following derivatives:

$$
\begin{gathered}
\nabla_{X} U=\phi H X, \\
\left(\nabla_{X} \phi\right) Y=f(Y) H X-\langle H X, Y\rangle U .
\end{gathered}
$$

We also have the usual Gauss and Codazzi equations for a real hypersurface of a complex space form (of holomorphic sectional curvature -4 ) in terms of $\phi$ and $H$ :

$$
\begin{gathered}
R(X, Y) Z=\langle X, Z\rangle Y-\langle Y, Z\rangle X+\langle\phi X, Z\rangle \phi Y-\langle\phi Y, Z\rangle \phi X \\
-2\langle X, \phi Y\rangle \phi Z+\langle H Y, Z\rangle H X-\langle H X, Z\rangle H Y \\
\left(\nabla_{X} H\right) Y-\left(\nabla_{Y} H\right) X=-f(X) \phi Y+f(Y) \phi X-2\langle X, \phi Y\rangle U
\end{gathered}
$$

for all $X, Y, Z \in T(M)$, where $R$ is the curvature tensor on $M$.

An important special case will be when $U$ is a principal direction on $M$. Under this assumption, more information can be gained concerning the structure of $M$. For example, it is straightforward to show that the direction $U$ is principal on $M$ if and only if the trajectories of $U$ on $M$ are geodesic [12]. In particular, the assumption that $U$ is a principal direction will force a strong relationship to hold between $H$ and $\phi$ :

Lemma 1 [12]. Suppose that $U$ is a principal direction on $M$ with principal curvature $\alpha$. Then

$$
2(H \phi H+\phi)=\alpha(\phi H+H \phi) \quad \text { in } T(M) .
$$

Since $\operatorname{ker}(f)$ is $\phi$-invariant (formulae (1.3)-(1.7)), it is natural to investigate the action of $\phi$ on $\operatorname{ker}(f)$.

Lemma 2 [12]. If $\lambda$ is a principal curvature on $M$, let $D_{\lambda}$ denote the distribution of principal directions on $M$ with principal curvature $\lambda$. If $\lambda^{2}-1 \neq 0$ on a neighborhood in $M$ and $X \in D_{\lambda} \cap \operatorname{ker}(f)$ on that neighborhood, then $\phi X$ is principal on that neighborhood.

Some information can be gleaned from the case that remains in the wake of Lemma 2:

Let $p \in M$ be a point at which $\lambda^{2}=1$ and $\lambda$ is a principal curvature with a direction $X \in(\operatorname{ker}(f))_{p}$. We may choose $\alpha=2$ at $p$ by selecting a suitable orientation of $M$. Assume that $\lambda=-1$ at $p$. Then, for $X \in\left(D_{\lambda}\right)_{p},(1.13)$ yields $\phi X$ principal with curvature 1 . However, if $\alpha=2$ and $\lambda=1$ at $p$, then 
for $X \in\left(D_{\lambda}\right)_{p},(1.13)$ is an identity and no information is gained concerning $\phi X$. This case must be treated separately.

We also note that assuming (1.13), $\lambda^{2} \neq 1, \alpha \neq \pm 2$ and $\alpha \neq 2 \lambda$ are equivalent statements.

The hypersurfaces studied in the sequel sections will have $U$ principal and constant principal curvatures. If $M$ is such a hypersurface with $\alpha \neq \pm 2$, we can choose a frame $\left\{X_{1}, \ldots, X_{n-1}, \phi X_{1}, \ldots, \phi X_{n-1}, U\right\}$ on $M$ that consists entirely of principal directions, with the property that $\phi$ interchanges the distributions $\operatorname{span}\left\{X_{1}, \ldots, X_{n-1}\right\}$ and $\operatorname{span}\left\{\phi X_{1}, \ldots, \phi X_{n-1}\right\}$. In this case $\phi$ acts on $\operatorname{ker}(f)$ as a complex structure and, from (1.13), the principal curvatures of a $\phi$-invariant plane $\operatorname{span}\left\{X_{i}, \phi X_{i}\right\}$ will be related by the equation

$$
\gamma_{i}=\left(\alpha \lambda_{i}-2\right) /\left(2 \lambda_{i}-\alpha\right)
$$

where $H X_{i}=\lambda_{i} X_{i}$ and $H \phi X_{i}=\gamma_{i} \phi X_{i}$.

\section{SOME FAMILIES OF ISOPARAMETRIC HYPERSURFACES}

In this section, families of isoparametric hypersurfaces in $\mathrm{CH}^{n}(-4)$ are constructed that have the distinguished direction $U$ as a principal direction. Our initial discussion will be of a more general nature: tubes in Riemannian manifolds. (For more detail, see $[1,4,7,9,18]$.)

Recall first the notions of cut point and cut locus. (A detailed and analytic discussion of cut loci can be found in Vol. II of [10] and in [11].) A cut point of a point $p$ in a Riemannian manifold $M$ is a point $c=\gamma(t)$, where $\gamma$ is a geodesic emanating from $p=\gamma(0)$ with the property that for $s>t$, the length of the curve $\gamma(J), J=[0, s]$, is greater than the distance $d_{M}(p, \gamma(s))$. For instance, if $p \in S^{2}(r)$, its only cut point is its antipodal point.

The cut locus of a point $p \in M$, written $\operatorname{Cut}(p)$, is the set of all cut points of $p$. The cut locus of a point on a sphere is a singleton, whereas for a point $p$ on a cylinder over $S^{1}$ in $\mathbf{R}^{3}, \operatorname{Cut}(p)$ is the axial line opposite $p$. Define $c(p)=\min \{d(p, q) \mid q \in \operatorname{Cut}(p)\}$.

Let $N^{m}$ be an immersed submanifold of a Riemannian manifold $M^{n}$. Define the unit normal sphere bundle of $N$ by

$$
S^{\perp}(N)=\left\{X \in T(N)^{\perp}:\|X\|=1\right\} .
$$

Set $c(N)=\inf \{c(p) \mid p \in N\}$. Now for each $r \in(0, c(N))$, define the tube of radius $r$ about $N$ in $M$ to be the hypersurface given by

$$
N_{r}=\left\{\exp _{q}(r X): q \in N, X \in S^{1}(N)\right\} .
$$

Let $\tau\left(t, X_{q}\right)$ be parallel translation of vector fields along the geodesic $\left(\gamma_{X}\right)_{q}$ : $t \rightarrow \exp _{q}(t X)$. For $p=\exp _{q}(r X) \in N_{r}, \tau\left(t, X_{q}\right): T_{q}(M) \rightarrow T_{p}(M)$ is a linear isometry $[15$, p. 66]. (See Figure 1.) By the generalized Gauss lemma [11, p. 121], we have

$$
T_{p}\left(N_{r}\right)=\tau\left(r, X_{q}\right)\left(\left\{X_{q}\right\}^{\perp}\right)=\left\{\tau\left(r, X_{q}\right) X_{q}\right\}^{\perp}
$$


and

$$
T_{p}\left(N_{r}\right) \cong T_{q}(N) \oplus\left[\left\{X_{q}\right\}^{\perp} \cap T_{q}(N)^{\perp}\right],
$$

where $\cong$ denotes the isomorphism of parallel translation. by

For $X \in S^{\perp}(N)$ and $q \in N$, define $\widetilde{R}_{X}(t) \in \operatorname{End}\left[T_{q}(N)\right]$, for each $t>0$,

$$
\tilde{R}_{X}(t) Y_{q}=\tau\left(t, X_{q}\right)^{-1}\left\{R\left(\tau\left(t, X_{q}\right) Y_{q}, \tau\left(t, X_{q}\right) X_{q}\right) \tau\left(t, X_{q}\right) X_{q}\right\}
$$

where $R$ is the curvature tensor of $M$. As we are primarily interested in the tangent space of the tube $N_{r}$, set

$$
R_{X}(t)=\left.\widetilde{R}_{X}(t)\right|_{\{X\}^{\perp}} \text {. }
$$

Finally, define $F(t, X) \in \operatorname{End}\left(\{X\}^{\perp}\right)$ for each $X \in S^{\perp}(N)$ to be the solution of the initial value problem

$$
\begin{gathered}
\left(d^{2} / d t^{2}\right)\left[F\left(t, X_{q}\right)\right]+R_{X}(t) \circ F\left(t, X_{q}\right)=0, \\
F\left(0, X_{q}\right)=P, \quad(d / d t)\left[F\left(t, X_{q}\right)\right]_{t=0}=-A_{X} \circ P+P^{\perp},
\end{gathered}
$$

for each $q \in N$, where $P:\{X\}^{\perp} \rightarrow T(N)$ and $P^{\perp}:\{X\}^{\perp} \rightarrow T(N)^{\perp} \cap\{X\}^{\perp}$ are orthogonal projections of the vector bundle $\{X\}^{\perp}=T(N) \oplus\left[T(N)^{\perp} \cap\right.$ $\{X\}^{\perp}$ ] onto the indicated component distributions, and $A_{X}$ is the Weingarten map of $X$ on $N$ in $M$. To simplify notation we shall write $F^{\prime}\left(s, X_{q}\right)$ for $\left.(d / d t)\left[F\left(t, X_{q}\right)\right]\right|_{t=s}$ and $F^{\prime \prime}\left(s, X_{q}\right)$ for $\left.\left(d^{2} / d t^{2}\right)\left[F\left(t, X_{q}\right)\right]\right|_{t=s}$.

Theorem $1[4,9,16]$. The second fundamental form of $N_{r}$ at $p=\exp _{q}(r X)$ is given by

$$
H_{r}=\tau\left(t, X_{q}\right) \circ F^{\prime}\left(r, X_{q}\right) \circ F\left(r, X_{q}\right)^{-1} \circ \tau\left(r, X_{q}\right)^{-1}
$$

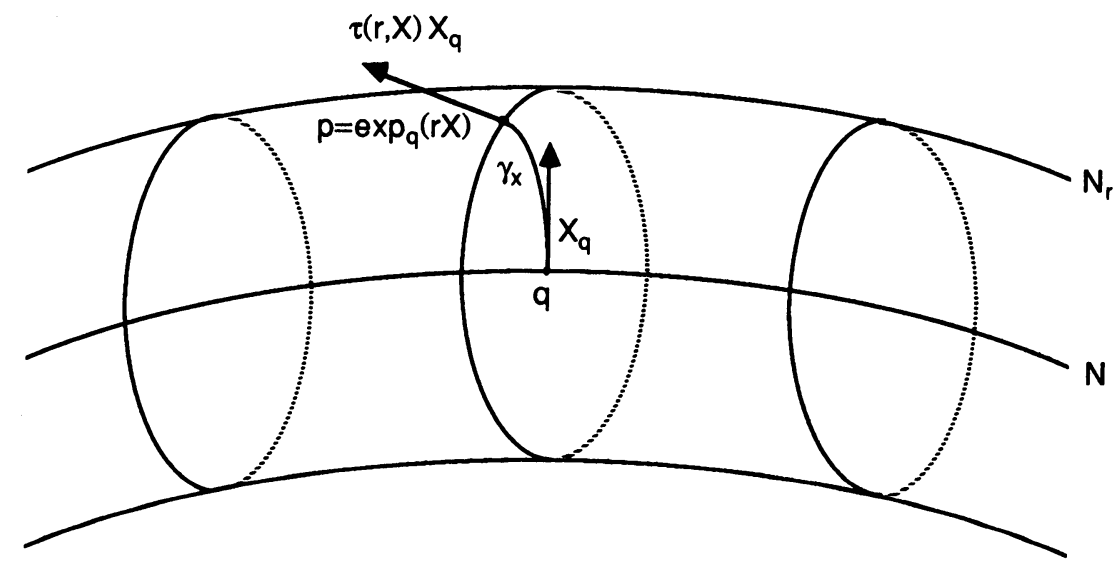

Figure 1 
Hence, in order to find an explicit representation of the second fundamental form of a tube, we need merely select a suitable basis of $T\left(N_{r}\right)$ using (2.1) and (2.2), solve (2.3), and then compute (2.4). Of course (2.4) says that $H_{r} \in$ $\operatorname{End}\left[T\left(N_{r}\right)\right]$ at $p=\exp _{q}(r X)$ is nothing more than parallel displacement of the endomorphism

$$
F^{\prime}\left(r, X_{q}\right) \circ F\left(r, X_{q}\right)^{-1} \in \operatorname{End}\left[\left\{X_{q}\right\}^{\perp}\right]
$$

along the geodesic $\gamma_{X}$ emanating from $q$ and passing through $p$.

Now we will assume that $M$ is not an arbitrary Riemannian manifold but the ambient space discussed in the previous section, namely a complex hyperbolic space. Let $N$ be an immersed orientable submanifold of $\mathbf{C} H^{n}(-4)$. As $\mathbf{C H}^{n}$ is a symmetric space, once a suitable basis of $\left\{X_{q}\right\}^{\perp}$ is selected (where $q \in N$ and $\left.X \in S^{\perp}(N)\right)$, parallel displacement along the geodesic $\gamma_{X}$ will preserve the basis and the respective orthogonality relations between its elements. Thus, $H_{r}$ will have the same matrix representation with respect to the displaced basis as $F^{\prime}\left(r, X_{q}\right) \circ F\left(r, X_{q}\right)^{-1}$ has with respect to the chosen basis of $\left\{X_{q}\right\}^{\perp}$. This simplifies the calculation of (2.4) considerably.

An additional feature of $\mathrm{CH}^{n}$ is that $R_{X}(r)$ is of a particularly simple form. Let $X \in S^{\perp}(N)$ and $Y \in\{X, J X\}^{\perp}$. Direct computations using the Gauss equation show that $\left(R_{X}(t) Y\right)_{q}=-Y_{q}$ and $\left(R_{X}(t) J X\right)_{q}=-4 J X_{q}$ for all $t>0$.

As $N_{r}$ is orientable, let $\xi$ be a local unit normal field on $N_{r}$. From the earlier discussion on tubes, at any point $p=\exp _{q}(r X) \in N_{r}$, we can write $\xi_{p}=\tau\left(r, X_{q}\right) X_{q}$. In this way a unique point $q$ and a unique direction in $S^{\perp}$ $\left(N_{q}\right)$ can be associated to each point $p \in N_{r}$.

In order to simplify notation, set $Y^{*}=\tau\left(r, X_{q}\right) Y \in T_{p}\left(N_{r}\right)$ for any $Y \in$ $\left\{X_{q}\right\}^{\perp}$. In particular, we shall write $\xi^{*}$ for a normal field on $N_{r}$, and $\xi$ will refer to the associated direction in $S^{\perp}(N)$; that is, $\xi_{p}^{*}=\exp _{q}\left(r \xi_{q}\right)$. In terms of $\S 1, U_{p}^{*}=-J \xi_{p}^{*}=\tau\left(r, X_{q}\right) J \xi_{q}$ and

$$
\left\{U_{p}^{*}\right\}^{\perp} \cap T_{p}\left(N_{r}\right)=\tau\left(r, \xi_{q}\right)\left[\left(T_{q}(N) \cap\left(J \xi_{q}\right)^{\perp}\right) \oplus\left(\left\{J \xi_{q}, \xi_{q}\right\}^{\perp} \cap T_{q}(N)^{\perp}\right)\right]
$$

is the $\phi$-invariant subspace, $(\operatorname{ker}(f))_{p}$, of $T_{p}\left(N_{r}\right)$.

Also, $\mathrm{CH}^{n}(-4)$, as a space of constant negative holomorphic sectional curvature, is a space of negative sectional curvature. Since $\mathrm{CH}^{n}(-4)$ is simply connected, by Theorem 8.1, Chapter VIII, Vol. 2 of [10], all cut loci will be empty. This means that tubes of radius less than $\min \{d(p, N): p$ a focal point of $N$ \} may be constructed about $N$.

The following are examples of tubes that form families of isoparametric hypersurfaces of the type discussed in $\S 1$. In each, it will be crucial that $U_{q}=\tau\left(r, X_{q}\right)^{-1} U_{p}^{*}$ is tangent or normal to the core at $q$. (Also see [1].)

Example 1. Let $N=H^{n}(-1)$ be a real space form of constant sectional curvature -1 immersed in $\mathrm{CH}^{n}$ as a totally geodesic and totally real submanifold. (See the proof of Theorem 1 in [3].) As $N$ has no focal points, for 


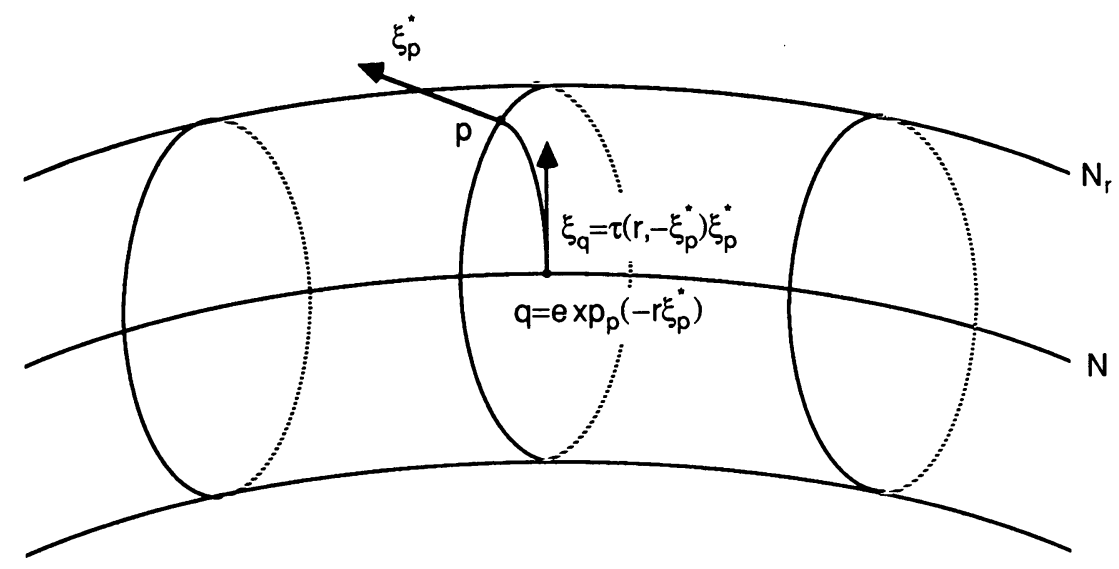

FIGURE 2

any $r>0$ let $N_{r}$ be the tube of radius $r$ about $N$ in $\mathrm{CH}^{n}$. Since $N$ is a totally real submanifold of dimension $n$, if $\xi^{*}$ is a unit normal to $N_{r}$, at each point $p \in \exp _{q}(r \xi) \in N_{r}$ we have $U_{q}=-J \xi_{q} \in T_{q}(N)$. So let $\left\{X_{1}, X_{2}, \ldots, X_{n-1}, U_{q}\right\}$ be an orthonormal basis of $T_{q}(N)$. If $(\phi, f)$ is the almost contact structure induced by $J$ on $N_{r}$, then

$$
B_{p}=\left\{X_{1}^{*}, \ldots, X_{n-1}^{*}, \phi\left(X_{1}^{*}\right), \ldots, \phi\left(X_{n-1}^{*}\right), U_{p}^{*}\right\}
$$

forms an orthonormal basis of $T_{p}\left(N_{r}\right)$. Setting $\phi X_{i}=\tau\left(r, \xi_{q}\right)^{-1}\left[\phi\left(X_{i}^{*}\right)\right]$ for each $i=1, \ldots, n-1$ allows us to write

$$
B_{q}=\left\{X_{1}, \ldots, X_{n-1}, \phi X_{1}, \ldots, \phi X_{n-1}, U_{q}\right\}
$$

for an orthonormal basis of $\left\{\xi_{q}\right\}^{\perp}$. With respect to this basis, (2.5) has the matrix solution

$$
F\left(t, \xi_{q}\right)=\operatorname{diag}\left(\cosh (t) I_{n-1}, \sinh (t) I_{n-1}, \cosh (2 t)\right) .
$$

Now, from (2.4), the second fundamental form, $H_{r}$, of the tube will have the following matrix representation with respect to the basis $B_{p}$ :

$$
H_{r}=\operatorname{diag}\left(\tanh (r) I_{n-1}, \operatorname{coth}(r) I_{n-1}, 2 \tanh (2 r)\right) .
$$

We see that the representation of $H_{r}$ depends only on $r$ and is hence constant on $N_{r}$. As a consequence $N_{r}$ is isoparametric. Also notice that $U$ is principal on $N_{r}$.

Example 2. Let $N=\mathbf{C} H^{k}, k=0,1, \ldots, n-1$, be a complex space form immersed in $\mathrm{CH}^{n}(-4)$ as a totally geodesic submanifold (see [3]). In case $k=0$, we are regarding a point to be a trivial complex space form. Otherwise, from [3], the $\mathrm{CH}^{k}$ will have constant holomorphic sectional curvature -4 . Again, as $N$ has no focal points, for any $r>0$ let $N_{r}$ be the tube of radius $r$ about $N$ in $\mathrm{CH}^{n}(-4)$ and $\xi^{*}$, be a global unit normal to $N_{r}$. As $N$ is 
a complex submanifold of $\mathbf{C H}^{n}$, its tangent space is invariant under $J$. So $\tau\left(r, \xi_{q}\right)\left[T_{q}(N)\right]$ is a $\phi$-invariant subspace of $T_{p}\left(N_{r}\right)$ and

$$
U_{q}=-\tau\left(r, \xi_{q}\right)^{-1} J \xi^{*} \in T_{q}^{\perp}(N) .
$$

Let $\left\{X_{1}, \ldots, X_{k}, J X_{1}, \ldots, J X_{k}\right\}$ be an orthonormal basis of $T_{q}(N)$ and extend to an orthonormal basis

$$
B_{q}=\left\{X_{1}, \ldots, X_{k}, J X_{1}, \ldots, J X_{k}, X_{k+1}, \ldots, X_{n-1}, J X_{k+1}, \ldots, J X_{n-1}, U_{q}\right\}
$$

of $\left\{\xi_{q}\right\}^{\perp}$. The last $2 n-1-2 k$ elements of $B_{q}$ form a basis of $T_{q}(N)^{\perp} \cap\left\{\xi_{q}\right\}^{\perp}$. The radially symmetric matrix solution to $(2.5)$, with respect to $B_{q}$, has the matrix representation

$$
F\left(t, \xi_{q}\right)=\operatorname{diag}\left(\cosh (t) I_{2 k}, \sinh (r) I_{2 n-2 k-2}, \sinh (2 r)\right) .
$$

Computing (2.4) yields the following matrix representation for the second fundamental form on $N_{r}$ with respect to the translation of $B_{q}$ :

$$
H_{r}=\operatorname{diag}\left(\tanh (r) I_{2 k}, \operatorname{coth}(r) I_{2 n-2 k-2}, 2 \operatorname{coth}(2 r)\right) \text {. }
$$

Again, $U^{*}=-J \xi^{*}$ is principal on $N_{r}$ and $N_{r}$ is isoparametric.

Example 3. The previous two examples, despite their obvious differences as tubes with different cores, do have one thing in common. Each core has no focal points so that tubes of any radii may be constructed. Hence, if the basis of $T_{p}\left(N_{r}\right), p=\exp _{q}\left(r \xi_{q}\right)$, is chosen for each $r>0$ to be compatible (via $\tau$ ) with $B_{q}$, then we can discuss the limit of the tensor $H_{r}$, viewed as acting on $\operatorname{span}\left(B_{q}\right)$, as $r \rightarrow \infty$. Clearly, this matrix limit is given by

$$
\lim _{r \rightarrow \infty} H_{r}=\operatorname{diag}\left(I_{2 n-2}, 2\right) \text {. }
$$

The geometric significance of this is obscure from our view of these tensors as acting on the tangent space of the core of the tube. However, in $\S 4$ (originally in [13]), a hypersurface is constructed in an analytic way that has a second fundamental form of the form (2.8). So we know that such a hypersurface exists; the question is whether we can obtain a geometric characterization.

Choose a point $p \in \mathbf{C} H^{n}$ and any direction $\xi \in T\left(\mathbf{C H}^{n}\right)$. For each $r>0$, let $q(r)=\exp _{p}\left(r \xi_{p}\right)$ and $\gamma_{\xi}$ be geodesic with initial direction $\xi_{p}$ that joins $p$ to $q(r)$. Then $p$ is on each geodesic hypersphere, $G_{r}(q(r))$, centered at $q(r)$ with radius $r$.

It is known that as $q(r)$ recedes from $p \quad(r \rightarrow \infty)$ the $G_{r}(q(r))$ approach a limiting hypersurface, $M^{\infty}$, called a horosphere (see $\left.[5,8]\right)$.

The horosphere will have an extrinsic geometry that is obtained as a limiting hypersurface of these expanding geodesic hyperspheres. That is, $M^{\infty}$ will have a second fundamental form with a representation (2.8) with respect to a suitable basis of $T_{p}\left(M^{\infty}\right)$. We shall establish this analytically in $\S 4$ when, subsequent to more theory, we will be able to show the convergence of the geodesic 


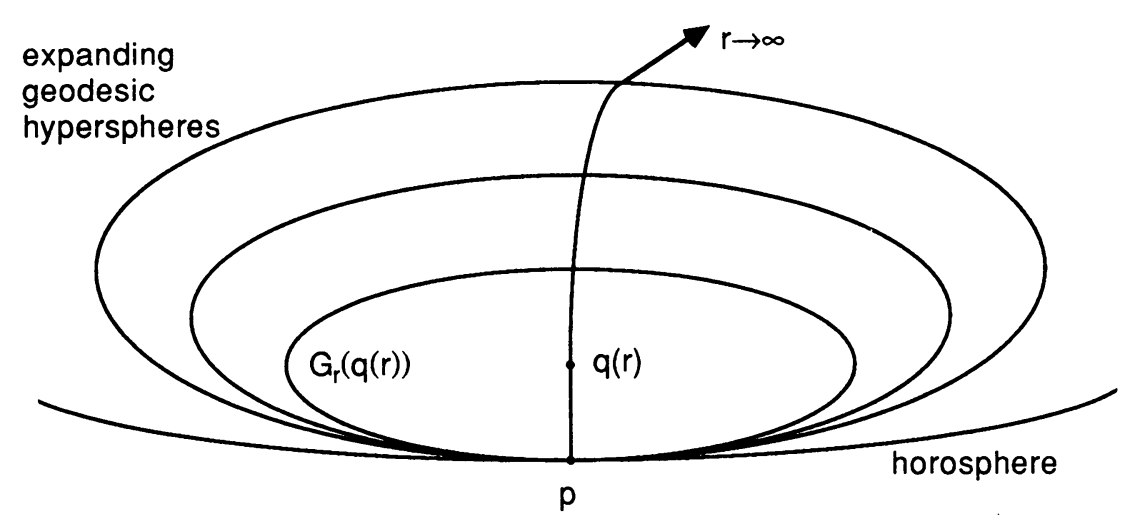

FIGURE 3

hyperspheres to a hypersurface with a second fundamental form of the type (2.8).

The hypersurfaces of Examples 1, 2, and 3 will yield satisfying geometric characterizations for isoparametric hypersurfaces of $\mathbf{C H}^{n}$ that satisfy a number of conditions. However, to make further progress in this direction we must show that hypersurfaces of this type are completely determined (up to congruences of $\mathrm{CH}^{n}$ ) by their second fundamental forms. In order to do this, $\mathrm{CH}^{n}$ must be thought of as the base manifold of a certain Lorentzian $S^{1}$-fiber bundle.

\section{THE LORENTZIAN CIRCLE BUNDLE OVER $\mathrm{CH}^{n}(-4)$ AND RIGIDITY OF CERTAIN ISOPARAMETRIC HYPERSURFACES OF $\mathrm{CH}^{n}(-4)$}

The best understood of all non-Euclidean complex space forms, complex projective space (usually written $\mathbf{C} P^{n}$ ), is constructed using a natural equivalence on an odd-dimensional sphere, $S^{2 n+1}$, itself immersed in $\mathbf{C}^{n+1}$. A complex hyperbolic space can be constructed in a similar way (see $[2 ; 10$, vol. II]). In this case $\mathrm{CH}^{n}$ is formed by taking the equivalence on a real Lorentzian hyperbolic space form in $\mathbf{C}^{n+1}$ instead of on a real Riemannian space form.

Define a hermitian bilinear form $F$ on $\mathbf{C}^{n+1}$ by

$$
F(z, w)=-z_{0} \bar{w}_{0}+\sum_{j=1}^{n} z_{j} \bar{w}_{j}
$$

for all $z=\left(z_{0}, z_{1}, \ldots, z_{n}\right)$ and $w=\left(w_{0}, w_{1}, \ldots, w_{n}\right)$ in $\mathbf{C}^{n+1} . F$ forms a complex Lorentzian metric, so that $\mathbf{C}^{n+1}$ equipped with the metric $F$ forms a complex Lorentzian space, $\mathbf{C}_{1}^{n+1}$, where at the origin $\left(z_{0}, 0, \ldots, 0\right)$ is a timelike vector if $z_{0} \neq 0 . \mathbf{C}^{n+1}$ can also be regarded as a real semi-Riemannian euclidean space, $\mathbf{R}_{2}^{2 n+2}$, if it is equipped with the metric $\operatorname{Re}(F)$. (At the origin $(1,0, \ldots, 0)$ and $(i, 0, \ldots, 0)$ form a basis of the negative definite subspace of $T_{0}\left(\mathbf{R}_{2}^{2 n+2}\right)$, with respect to the metric $\operatorname{Re}(F)$.) Anti-De Sitter spacetime is 
the hyperquadric defined by

$$
H_{1}^{2 n+1}=\left\{z \in \mathbf{C}^{n+1} \mid F(z, z)=-1\right\} .
$$

The tangent space of anti-De Sitter space is determined by its immersion into the ambient complex euclidean space; explicitly:

$$
T_{z}\left(H_{1}^{2 n+1}\right)=\left\{w \in \mathbf{C}^{n+1} \mid \operatorname{Re}[F(z, w)]=0\right\}
$$

for any $z \in H_{1}^{2 n+1}$. As a real hypersurface of $\mathbf{R}_{2}^{2 n+2}, H_{1}^{2 n+1}$ has $\left.\operatorname{Re}(F)\right|_{H_{1}^{2 n+1}}$ as a natural Lorentzian metric that is of constant sectional curvature -1 .

An $S^{1}$-action can be defined on $H_{1}^{2 n+1}$ (in fact on $\mathbf{C}_{1}^{n+1}$ ) by $z \rightarrow \lambda z$ for any $z \in H_{1}^{2 n+1}$ and $\lambda \in \mathbf{C}$ with $|\lambda|=1$. At each point $z \in H_{1}^{2 n+1}$, the vector $V=i z$ is tangent to the flow and has length -1 . Given a point $z \in H_{1}^{2 n+1}$, the flow of $V$ through $z$ in $H_{1}^{2 n+1}$ will be given by the orbit

$$
O_{z}=\left\{x_{t}=e^{i t} z \mid t \in \mathbf{R}\right\}
$$

that satisfies the differential equation $d x_{t} / d t=i x_{t}$. This in turn shows that $O_{z}$ lies in the intersection of the negative definite plane $\operatorname{span}\{z, V\}$ with $H_{1}^{2 n+1}$. Let $\sim$ be the equivalence given by the orbits of the action, that is, $w \sim z$ if $w \in O_{z}$. Then the natural projection $\pi: H_{1}^{2 n+1} \rightarrow H_{1}^{2 n+1} / \sim=\mathbf{C} H^{n}$ is a Riemannian submersion with fundamental tensor the natural complex structure $J$ on $\mathbf{C}_{1}^{n+1}$ (see [13, 16]) and with timelike totally geodesic fibers, each of which is a trajectory of the vertical vector $V=i z$ at any point $z \in H_{1}^{2 n+1}$. The complex Riemannian space, $\mathrm{CH}^{n}$, obtained in this way has its complex structure induced from that on $\mathbf{C}_{1}^{n+1}$ and has constant holomorphic sectional curvature -4 with the metric induced from $\operatorname{Re}(F)$.

The differential of the submersion, $\pi_{*}$, is a linear isometry [16]; i.e., it preserves the metric tensor on the horizontal distribution as it projects onto $T\left(\mathrm{CH}^{n}\right)$. So we shall make no distinction between the metric on $H_{1}^{2 n+1}$ and that on $\mathrm{CH}^{n}$. We can now write

$$
T_{z}\left(H_{1}^{2 n+1}\right) \cong T_{\pi(z)}\left(\mathbf{C} H^{n}\right) \oplus \operatorname{span}\{V\}
$$

where the isomorphism is given by $\left.\pi_{*} \otimes I\right|_{\operatorname{span}\{V\}}$.

$\pi_{*}$, as a linear isometry of the distribution $\mathscr{H}$ of horizontal vector fields on $H_{1}^{2 n+1}$ onto $T\left(\mathrm{CH}^{n}\right)$, induces relations between the connections $\dot{\nabla}$ and $\bar{\nabla}$. of $H_{1}^{2 n+1}$ and $\mathrm{CH}^{n}$, respectively. (See $[6,16]$.) These are

$$
\begin{aligned}
& \dot{\nabla}_{\left(X^{\sim}\right)} Y^{\sim}=\left(\bar{\nabla}_{X} Y\right)^{\sim}+\left\langle J\left(X^{\sim}\right), Y^{\sim}\right\rangle V, \\
& \dot{\nabla}_{V}\left(X^{\sim}\right)=\dot{\nabla}_{\left(X^{\sim}\right)} V=(J X)^{\sim}=J\left(X^{\sim}\right)
\end{aligned}
$$

for all $X, Y \in T\left(\mathbf{C} H^{n}\right) \cong \mathscr{H}$, and where $X^{\sim}$ denotes the unique horizontal lift of a vector field $X \in T\left(\mathbf{C} H^{n}\right)$. 
If $M^{2 n-1}$ is an orientable real hypersurface of $\mathrm{CH}^{n}(-4)$ then the hypersurface $\widetilde{M}_{1}^{2 n}=\pi^{-1}(M)$ of $H_{1}^{2 n+1}$ is orientable and invariant under the $S^{1}$ action, and $\left.\pi\right|_{\widetilde{M}}: \widetilde{M} \rightarrow M$ is a Riemannian submersion with timelike totally geodesic fibers. Conversely, if $\widetilde{M}_{1}^{2 n}$ is an orientable $S^{1}$-invariant hypersurface of $H_{1}^{2 n+1}$, then $\left.\pi\right|_{\widetilde{M}}$ is a Riemannian submersion of $\widetilde{M}$ onto $M^{2 n-1}=\pi(\widetilde{M})$ with timelike totally geodesic fibers. Hence, we have the following commutative diagram:

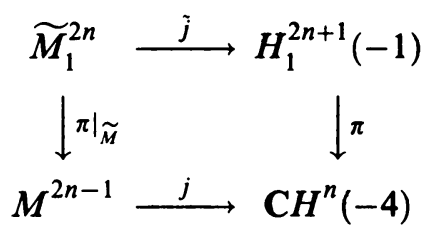

where $j: M^{2 n-1} \rightarrow \mathrm{C} H^{n}(-4)$ and $\tilde{j}: \widetilde{M}_{1}^{2 n} \rightarrow H_{1}^{2 n+1}(-1)$ are immersions compatible with the fibration. If $\xi$ is a normal field on $M$, its unique lift, $\xi^{\sim}$, is horizontal and forms a normal field on $\widetilde{M}$. Henceforth, we will drop this distinction between $\xi$ and $\xi^{\sim}$.

Let $\widetilde{\nabla}$ be the metric connection of $\widetilde{M}$ as a hypersurface in $H_{1}^{2 n+1}$ and $\widetilde{H}$ be its second fundamental form. The Gauss and Weingarten formulae in this case are given by

$$
\dot{\nabla}_{X} Y=\tilde{\nabla}_{X} Y+\langle\tilde{H} X, Y\rangle \xi \text { and } \dot{\nabla}_{X} \xi=-\tilde{H} X
$$

for all $X, Y \in T(\widetilde{M})$. Now combining (1.1), (3.1), (3.2), and (3.3) we have the following relations between $\widetilde{H}$ and $H$ :

$$
\widetilde{H}\left(X^{\sim}\right)=(H X)^{\sim}-f(X) V
$$

for all $X \in T(M) \cong\{V\}^{\perp} \cap T(\widetilde{M})$ and

$$
\tilde{H} V=U^{\sim} \text {. }
$$

Let $\widetilde{R}$ be the curvature tensor of $\widetilde{M}$. We shall have the opportunity to use the Gauss and Codazzi equations for $\widetilde{M}$ in $H_{1}^{2 n+1}$ :

$$
\begin{gathered}
\widetilde{R}(X, Y) Z=\langle X, Z\rangle Y-\langle Y, Z\rangle X+\langle\widetilde{H} Y, Z\rangle \tilde{H} X-\langle\tilde{H} X, Z\rangle \tilde{H} Y \\
\left(\tilde{\nabla}_{X} \widetilde{H}\right) Y=\left(\tilde{\nabla}_{Y} \widetilde{H}\right) X
\end{gathered}
$$

for all $X, Y \in T(\widetilde{M})$. These and the preceding formulae can be used to prove the following useful identities:

$$
\begin{aligned}
\left(\tilde{\nabla}_{W} \widetilde{R}\right)(X, Y) Z= & \left\langle\left(\widetilde{\nabla}_{W} \widetilde{H}\right) Y, Z\right\rangle \widetilde{H} X-\left\langle\left(\tilde{\nabla}_{W} \widetilde{H}\right) X, Z\right\rangle \widetilde{H} Y \\
& +\langle\widetilde{H} Y, Z\rangle\left(\tilde{\nabla}_{W} \tilde{H}\right) X-\langle\widetilde{H} X, Z\rangle\left(\tilde{\nabla}_{W} \widetilde{H}\right) Y
\end{aligned}
$$

for all $X, Y, Z, W \in T(\widetilde{M})$, and

$$
\left(\widetilde{\nabla}_{\left(X^{\sim}\right)} \widetilde{H}\right) V=[(\phi H-H \phi) X]^{\sim}
$$

for any $X \in T(M)$. 
An immediate consequence of the preceding formulae is that $M$ and $\widetilde{M}$ have the same mean curvatures. Thus, $\widetilde{M}$ is isoparametric when $M$ is isoparametric.

If $\left\{X_{1}, \ldots, X_{2 n-1}\right\}$ is a local frame on $M$ consisting of principal directions in $T(M)$ with corresponding principal curvatures $\left\{\lambda_{1}, \ldots, \lambda_{2 n-1}\right\}$, then $\left\{X_{1}^{\sim}, \ldots, X_{2 n-1}^{\sim}, V\right\}$ forms a local frame on $\widetilde{M}$ with respect to which $\widetilde{H}$ is represented by the matrix

$$
\left[\begin{array}{rrcc}
\lambda_{1} & & & f\left(X_{1}\right) \\
& & 0 & \vdots \\
& 0 & \lambda_{2 n-1} & f\left(X_{2 n-1}\right) \\
-f\left(X_{1}\right) & \cdots & -f\left(X_{2 n-1}\right) & 0
\end{array}\right],
$$

all of whose entries are functions on $\widetilde{M}$. Hence, even the stipulation that $\widetilde{M}$ be $S^{1}$-invariant does not guarantee an easy analysis of the structure of $\widetilde{M}$ via an investigation of those subbundles of $T(\widetilde{M})$ held invariant by $\widetilde{H}$, for we are not even assured the existence of principal directions that have real principal curvatures.

However, in case $U$ is a principal direction on $M$, we can choose $U=X_{2 n-1}$ so that $\widetilde{H}$ is represented by the matrix

$$
\operatorname{diag}\left(\lambda_{1}, \ldots, \lambda_{2 n-2},\left[\begin{array}{cc}
\alpha & 1 \\
-1 & 0
\end{array}\right]\right) \text {. }
$$

We shall see that if $M$ is also isoparametric, then $M$ is rigid; that is, $M$ is unique up to congruence.

In order to characterize congruence in $\mathrm{CH}^{n}(-4)$, the principal $S^{1}$-fiber bundle over $\mathrm{CH}^{n}$ must be taken into account as well as the fiber bundle's own imbedding in $\mathrm{C}_{1}^{n+1}$. From the preceding discussion we see that rigid motions of $\mathrm{C}_{1}^{n+1}$ that leave $H_{1}^{2 n+1}$ invariant will induce rigid motions of $\mathbf{C} H^{n}$.

The isometries of $\mathbf{C}_{1}^{n+1}$ under $F$,

$$
U(1, n)=\left\{A \in G L(n+1 ; \mathbf{C}): F(A z, A w)=F(z, w), \forall z, w \in \mathbf{C}_{1}^{n+1}\right\},
$$

are precisely the rigid motions that leave $H_{1}^{2 n+1}$ invariant and act transitively on $H_{1}^{2 n+1}$. Hence, the elements of $U(1, n)$ will induce isometries of $\mathrm{CH}^{n}$ via $\pi$.

Now let $M$ and $N$ be isoparametric hypersurfaces of $\mathrm{CH}^{n}$ that each has the distinguished direction of the induced almost contact structure as a principal direction. Suppose that $M$ and $N$ have second fundamental forms with the same matrix representation with respect to suitably chosen orientations of $M$ and $N$ and local orthonormal bases of principal directions of $T(M)$ and $T(N)$. Let $\widehat{M}, \rho$ and $\widehat{N}, \sigma$ be the simply connected covering spaces of the lifts $\widetilde{M}=\pi^{-1}(M)$ and $\widetilde{N}=n^{-1}(N)$, respectively. Since $\widetilde{N}$ and $\widetilde{M}$ are isoparametric, following an argument in [17], combine (3.10) with the fact that 
the ambient space of $\widetilde{N}$ and $\widetilde{M}$ is of constant sectional curvature to show that the principal curvatures $\alpha$ and $\lambda_{i}, i=1, \ldots, 2 n-1$, are constant.

If $i: M \rightarrow \mathrm{CH}^{n}$ and $j: N \rightarrow \mathbf{C} H^{n}$ are the isometric immersions in complex hyperbolic space and $\tilde{i}: \widetilde{M} \rightarrow H_{1}^{2 n+1}$ and $\tilde{j}: \tilde{N} \rightarrow H_{1}^{2 n+1}$ are the induced immersions of the respective lifts, we have the following commutative diagram of immersions, submersions, and covering maps:

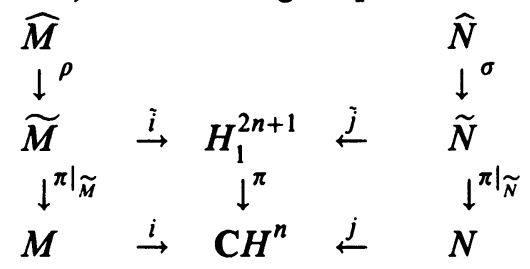

The maps $\tilde{i} \circ \rho: \widehat{M} \rightarrow H_{1}^{2 n+1}$ and $\tilde{j} \circ \sigma: \widehat{N} \rightarrow H_{1}^{2 n+1}$ are now isometric immersions of simply connected Lorentzian spaces of codimension 1 into the Lorentzian symmetric space $H_{1}^{2 n+1}$ that have the same constant matrix representation for their second fundamental forms with respect to canonically chosen orthonormal bases of $T(\widehat{M})$ and $T(\widehat{N})$.

Lemma 3. Let $N$ and $M$ be isoparametric oriented real hypersurfaces of $\mathrm{CH}^{n}(-4)$ that have

(i) $U$ as a principal direction, and

(ii) the same corresponding principal curvatures, i.e., the same matrix representation for the second fundamental form with respect to suitably chosen bases of corresponding principal directions.

If $\widehat{N}$ and $\widehat{M}$ are the simply connected covering spaces of $\tilde{N}=\pi^{-1}(N)$ and $\widetilde{M}=\pi^{-1}(M)$, respectively, then $\widehat{N}$ and $\widehat{M}$ are isometric.

Sketch of Proof. Let $x \in \widehat{N}$ and $y \in \widehat{M}$. By hypothesis and using the ideas of $\S 1$ we can choose orthonormal bases of $T_{\pi(\sigma(x))}(N)$ and $T_{\pi(\rho(y))}(M)$ that consist entirely of principal directions, with respect to which the second fundamental forms have the same representations. Now pull back and extend to orthonormal bases of $T_{x}(\widehat{N})$ and $T_{y}(\widehat{M})$. (These constructions rely primarily on the distinguished direction $U$ being principal on both $M$ and $N$.)

Define a linear isometry $\psi: T_{x}(\widehat{N}) \rightarrow T_{y}(\widehat{M})$ in the "obvious" way; that is, $\psi$ maps basis elements of $T_{x}(\widehat{N})$ onto the corresponding basis elements of $T_{y}(\widehat{M})$. Let $\widehat{H}$ and $\widehat{H}^{\prime}$ denote the second fundamental forms of the isometric immersions $\tilde{j} \circ \sigma$ and $\tilde{i} \circ \rho$, and similarly let $\tilde{H}$ and $\tilde{H}^{\prime}$ denote the second fundamental forms of the immersions $\tilde{j}$ and $\tilde{i}$, respectively. As $\sigma$ and $\rho$ are local isometries, $\widehat{H}_{x}$ and $\widehat{H}_{y}^{\prime}$ will agree with $\widetilde{H}_{\sigma(x)}$ and $\widetilde{H}_{\rho(y)}^{\prime}$, respectively. By hypothesis and applying (3.4) and (3.5) we have that $\psi\left(\widehat{H}_{x}(Z)\right)=\widehat{H}_{y}^{\prime}(\psi(Z))$ for any $Z \in T_{x}(\hat{N})$. Let $\widehat{R}$ and $\hat{R}^{\prime}$ denote the curvature tensors of $\widehat{N}$ and $\widehat{M}$, respectively. By (3.6), $\psi\left(\hat{R}_{x}(X, Y) Z\right)=\widehat{R}_{y}^{\prime}(\psi X, \psi Y) \psi Z$ for all $X, Y, Z \in$ $T_{x}(\widehat{N})$. 
Let $\hat{\nabla}$ and $\hat{\nabla}^{\prime}$ denote the connections on $\widehat{N}$ and $\widehat{M}$. Using (3.7) and (3.9), it follows that $\psi$ maps the tensor $(\hat{\nabla} \widehat{H})_{x}$ to the tensor $\left(\widehat{\nabla}^{\prime} \widehat{H}^{\prime}\right)_{y}$. Hence by (3.8), $\psi$ maps $(\hat{\nabla} \widehat{R})_{x}$ to $\left(\widehat{\nabla}^{\prime} \hat{R}^{\prime}\right)_{y}$. The fact that $\widehat{H}$ and $\widehat{H}^{\prime}$ have corresponding constant matrix representations provides the inductive step to show that $\psi$ maps $\left(\hat{\nabla}^{m} \widehat{R}\right)_{x}$ to $\left(\hat{\nabla}^{\prime m} \hat{R}^{\prime}\right)_{y}$ for any $m>0$.

Now by Corollary 7.3, Chapter VI, Vol. I of [10], there is a unique isometry $\Omega: \widehat{N} \rightarrow \widehat{M}$ such that $(d \Omega)_{x}=\psi$.

The point of Lemma 3 is to get into position to establish a general congruence theorem for real hypersurfaces of complex space forms. We shall establish this by using known congruence results for submanifolds of semi-Riemannian euclidean spaces.

Theorem 2. Let $M$ and $N$ be isoparametric orientable real hypersurfaces of $\mathrm{CH}^{n}$ that each have the distinguished direction $U$ as a principal direction. If $\mathbf{M}$ and $N$ have the same second fundamental form, i.e., the corresponding principal curvatures are the same, then $M$ and $N$ are congruent.

Sketch of the Proof. Regard the simply connected covering spaces, $\widehat{M}$ and $\widehat{N}$ of the lifts of $M$ and $N$ in $H_{1}^{2 n+1}$, as Lorentzian submanifolds of the semiRiemannian manifold $\mathbf{R}_{2}^{2 n+2}$. Define $\psi^{\perp}: T_{x}(\widehat{N})^{\perp} \subset T_{x}\left(\mathbf{R}_{2}^{2 n+2}\right) \rightarrow T_{y}(\widehat{M})^{\perp} \subset$ $T_{x}\left(\mathbf{R}_{2}^{2 n+2}\right)$ by $\psi^{\perp}(x)=y$ and $\psi^{\perp}\left(\xi_{x}\right)=\xi_{y}^{\prime}$, where $\xi$ and $\xi^{\prime}$ are the normals to $\widehat{N}$ and $\widehat{M}$ in $H_{1}^{2 n+1}$, and then extend linearly to obtain a linear isometry of the two-dimensional normal spaces. Utilize the hypotheses that $M$ and $N$ are isoparametric and have the direction $U$ principal and the fact that $H_{1}^{2 n+1}$ is a totally umbillic (and isoparametric) hyperquadric in $\mathbf{R}_{2}^{2 n+2}$ to show that for any curve $\alpha$ through $x$ in $\widehat{N}$, the linear isometry of normal spaces

$$
\psi_{\alpha(s)}^{\perp}=P_{\Omega(\alpha(s))} \circ \psi^{\perp} \circ P_{\alpha(s)}^{-1}: T_{\alpha(s)}(\widehat{N})^{\perp} \rightarrow T_{\Omega(\alpha(s))}(\widehat{M})^{\perp},
$$

where $P$ is normal parallel translation along smooth curves, maps the second fundamental form of $\widehat{N}$ at $\alpha(s)$ onto that of $\widehat{M}$ at $\Omega(\alpha(s))$, regarded as submanifolds of $\mathbf{R}_{2}^{2 n+2} \approx \mathbf{C}_{1}^{n+1}$.

Finally, use Lemma 3 and Theorem 41 , Chapter 4 of [15], to show that there exists an isometry $\bar{\psi}$ of $\mathbf{C}_{1}^{n+1}$ such that $\left.\bar{\psi}\right|_{\widehat{N}}=\Omega$. Hence, $\bar{\psi}$ induces a rigid motion of $\mathrm{CH}^{n}$ that maps $N$ isometrically onto $M$.

Theorem 2 will be used in the following section to establish the existence of a horosphere in $\mathrm{CH}^{n}$ independent of $[5,8,18]$. Theorem 2 will also be useful in the future for classifying real hypersurfaces of $\mathrm{CH}^{n}$ that satisfy certain tensor equations.

\section{APPLICATION: AN ANALYTIC CONSTRUCTION OF A HOROSPHERE}

Using the congruence results of $\S 3$, we can now place the model spaces used in [13] into the context of the hypersurfaces constructed in $\S 2$. First, recall 
an elegant and well-known (e.g., [13, 14]), analytic method of determining the extrinsic geometry of a level hypersurface of a $C^{\infty}$ function on a space form imbedded as a hypersurface in a euclidean space, modified to fit the particular needs of this section.

Let $f: \mathbf{R}_{2}^{n+1} \rightarrow \mathbf{R}$ be a $C^{\infty}$ function and $M_{1}^{n}(c)$ be an imbedded Lorentzian space form in $\mathbf{R}_{2}^{n+1}$ of sectional curvature $c$. Let $\nabla f$ denote the gradient of $f$ in $T(M)$ as a function on $M$, and $\tilde{\nabla} f$ denote the gradient of $f$ as a function on $\mathbf{R}_{2}^{n+1}$. Let $S$ be the set of all $s \in \mathbf{R}$ such that $\widetilde{M}_{s}=f^{-1}(s)$ is a hypersurface of $\mathbf{R}_{2}^{n+1}$. Then for any $s \in S, \widetilde{M}_{s}$ and $\tilde{\nabla} f /\|\tilde{\nabla} f\|$ as a unit normal field in $T\left(\mathbf{R}_{2}^{n+1}\right)$. Similarly, let $T$ be the set of all $s \in S$ such that $M_{s}=\widetilde{M}_{s} \cap M_{1}^{n}(c)$ is a hypersurface of $M_{1}^{n}(c)$. Then $M_{s}$ will have $\nabla f /\|\nabla f\|$ as a unit normal in $T\left(M_{1}^{n}(c)\right)$, for each $s \in T$.

For a given $f, \tilde{\nabla} f$ is usually easy to calculate. Once this is done,

$$
\nabla f=\tilde{\nabla} f+\langle\tilde{\nabla} f, \zeta\rangle \zeta
$$

where $\langle$,$\rangle is the standard metric of \mathbf{R}_{2}^{n+1}$ and $\zeta$ is a unit timelike normal field to $M_{1}^{n}(c)$ in $\mathbf{R}_{2}^{n+1}$. (Notice that the choice of positive coefficient of $\zeta$ is necessitated by the causal nature of $\zeta$.)

Let $\operatorname{Hess}\left(f ; \mathbf{R}_{2}^{n+1}\right)$ denote the hessian of $f$ as an operator on $\mathbf{R}_{2}^{n+1}$. For each $s \in S$, the second fundamental form $\widetilde{H}$ of $\widetilde{M}_{s}$ in $\mathbf{R}_{2}^{n+1}$ is given by

$$
\langle\widetilde{H} X, Y\rangle=\operatorname{Hess}\left(f ; \mathbf{R}_{2}^{n+1}\right)(X, Y) /\|\widetilde{\nabla} f\|
$$

for all $X, Y \in T\left(\widetilde{M}_{s}\right)$, which for a given $f$ is usually easy to compute. For each $s \in T$, the second fundamental form $H$ of $M_{s}$ in $M_{1}^{n}(c)$ can be expressed similarly:

$$
\langle H X, Y\rangle=\operatorname{Hess}\left(f ; M_{1}^{n}(c)\right)(X, Y) /\|\nabla f\|
$$

for all $X, Y \in T\left(M_{s}\right)$ and where $\operatorname{Hess}\left(f ; M_{1}^{n}(c)\right)$ denotes the hessian of $f$ as an operator on $T\left(M_{1}^{n}(c)\right)$. Since $\operatorname{Hess}\left(f ; M_{1}^{n}(c)\right)$ is defined to be the second covariant derivative of $f$ on $M_{1}^{n}(c)$ [15]:

$$
\operatorname{Hess}\left(f ; M_{1}^{n}(c)\right)(X, Y)=X Y f-\left(\nabla_{X} Y\right) f=\left\langle\nabla_{X}(\nabla f), Y\right\rangle \text {, }
$$

where $\nabla$ denotes the connection on $M_{1}^{n}(c)$; once (4.2) has been computed, (4.1) can be used to obtain a representation of (4.3), thereby yielding an explicit calculation of the second fundamental form of $M_{s}$ in $M_{1}^{n}(c)$.

Example 4. Consider the function $G_{p}: \mathbf{C}_{1}^{n+1} \rightarrow \mathbf{R}$, for each $p=0,1, \ldots, n$, defined by

$$
G_{p}(z)=-\left|z_{0}\right|^{2}+\sum_{i=1}^{p}\left|z_{i}\right|^{2}
$$

where $z=\left(z_{0}, z_{1}, \ldots, z_{n}\right)$. For $r>0$, define a level hypersurface of $\mathbf{C}_{1}^{n+1}$ by

$$
\widetilde{M}_{p}(r)=\left\{z \in \mathbf{C}_{1}^{n+1} \mid G_{p}(z)=-\cosh ^{2}(r)\right\} .
$$


The gradient of $G_{p}$ in $C_{1}^{n+1}$ is computed to be

$$
\widetilde{\nabla} G_{p}(z)=2\left(z_{0}, z_{1}, \ldots, z_{p}, 0, \ldots, 0\right)
$$

for all $z \in \mathbf{C}_{1}^{n+1}$, so that

$$
\tilde{\nabla} G_{p}(z) / 2 \cosh (r)=\left(\operatorname{sech}(r) z_{0}, \operatorname{sech}(r) z_{1}, \ldots, \operatorname{sech}(r) z_{p}, 0, \ldots, 0\right)
$$

is a unit (timelike) normal to $\widetilde{M}_{p}(r)$ in $\mathbf{C}_{1}^{n+1}$, for all $z \in \widetilde{M}_{p}(r)$.

The level hypersurface $M_{p}(r)=\widetilde{M}_{p}(r) \cap H_{1}^{2 n+1}$ is nothing more than the model $M_{2 p+1,2 q+1}\left(\tanh ^{2}(r)\right)$ of example 4.1 in [13]. Notice that $M_{p}(r)$ is isometric to the product $H_{1}^{2 p+1}\left(-\cosh ^{2}(r)\right) x S^{2(n-p)-1}\left(\sinh ^{2}(r)\right)$. The gradient of $G_{p}$ on $M_{p}(r)$ in $T\left(H_{1}^{2 n+1}\right)$ is given by (4.1)

$$
\nabla G_{p}(z)=-2 \cosh ^{2}(r)\left(\tanh ^{2}(r) z_{0}, \ldots, \tanh ^{2}(r) z_{p}, z_{p+1}, \ldots, z_{n}\right)
$$

for all $z \in M_{p}(r)$. Thus,

$$
\xi(z)=-\left(\tanh (r) z_{0}, \ldots, \tanh (z) z_{p}, \operatorname{coth}(r) z_{p+1}, \ldots, \operatorname{coth}(r) z_{n}\right)
$$

is a unit normal to $M_{p}(r)$ in $H_{1}^{2 n+1}$, for all $z \in M_{p}(r)$.

At this point we can see that the second fundamental form of $M_{p}(r)$ in $H_{1}^{2 n+1}$ is diagonalizable with respect to a real basis of $T_{z}\left(M_{p}(r)\right)=\{z, \xi(z)\}^{\perp}$ and has constant principal curvatures $\tanh (r)$ and $\operatorname{coth}(r)$ of real multiplicities $2 p+1$ and $2 n-2 p-1$, respectively.

Let $N_{p}(r)=\pi\left(M_{p}(r)\right)$ and $U$ denote the distinguished vector on $N_{p}(r)$ viewed as a real hypersurface of $\mathrm{CH}^{n}$. Let $H^{\prime}$ and $H$ denote the second fundamental forms of $M_{p}(r)$ and $N_{p}(r)$, respectively. We can write

$$
U_{\pi(z)}=-J\left(\pi_{*}(\xi(z))\right)=\pi_{*}(-i \xi(z)) \text {, i.e., }\left(U^{\sim}\right)_{z}=-i \xi(z) \text {. }
$$

An explicit calculation of $H^{\prime}\left(U^{\sim}\right)_{z}$ using (4.3) followed by an application of (3.4) shows that $U_{\pi(z)}$ is principal in $T_{\pi(z)}\left(N_{p}(r)\right)$ with curvature $2 \operatorname{coth}(2 r)$. Subsequent calculations yield the other principal curvatures $\tanh (r)$ and $\operatorname{coth}(r)$ of multiplicities $2 p$ and $2 n-2 p-2$, respectively, each having a $\phi$-invariant eigendistribution. From the work of $\S \S 2$ and 3 we see that $N_{p}(r)$ is congruent to a tube of radius $r$ about a totally geodesic complex space form in $\mathrm{CH}^{n}$ isometric to a $\mathrm{CH}^{p}$. In particular, we shall need that $N_{0}(r)$ is congruent to a geodesic hypersphere of radius $r$.

Example 5. Consider the function $G: \mathbf{C}_{1}^{n+1} \rightarrow \mathbf{R}$ given by $G(z)=\left|z_{0}-z_{1}\right|^{2}$, where $z=\left(z_{0}, \ldots, z_{n}\right)$, and the level hypersurface of $\mathbf{C}_{1}^{n+1}, \widetilde{M}=G^{-1}(1)$. The gradient of $G$ in $C_{1}^{n+1}$ can be written as

$$
\tilde{\nabla} G(z)=2\left(z_{1}-z_{0}, z_{1}-z_{0}, 0, \ldots, 0\right)
$$

for all $z \in \widetilde{M}$. 
The level hypersurface $N=\widetilde{M} \cap H_{1}^{2 n+1}$ is the model hypersurface $N$ of example 4.2 of [13]. The gradient of $G$ in $H_{1}^{2 n+1}$ is given by

$$
\nabla G(z)=2\left(z_{1}, 2 z_{1}-z_{0}, z_{2}, \ldots, z_{n}\right)
$$

for all $z \in N$. Hence,

$$
\xi(z)=\left(z_{1}, 2 z_{1}-z_{0}, z_{2}, \ldots, z_{n}\right)
$$

is a unit normal to $N$ in $H_{1}^{2 n+1}$.

Set $M_{n}^{*}=\pi(N)$, as in example 4.2 of [13]. Explicit calculations using (4.3) and (3.4) show that $U$ is principal on $M_{n}^{*}$ with principal curvature 2 and that 1 is a principal curvature of multiplicity $2 n-2$; that is, the second fundamental form of $M_{n}^{*}$ acts as the identity transformation on $\operatorname{ker}(f)$. Thus, according to Theorem 2, $M_{n}^{*}$ is our candidate for a horosphere. In order to see this (and thereby establish the existence of a horosphere in $\mathrm{CH}^{n}$ analytically) we will show that $M_{n}^{*}$ is a limiting hypersurface of a specific family of geodesic hyperspheres.

Let $P=(1,0, \ldots, 0) \in H_{1}^{2 n+1}$ and consider the geodesic emanating from $\pi(P)$ in $\mathrm{CH}^{n}$ given by $\gamma(r)=\pi(\cosh (r), \sinh (r), 0, \ldots, 0)$. (See [10, Vol. II, p. 285].) As in example 3, each geodesic hypersphere of radius $r$ centered at $\gamma(r)$ contains the point $\pi(P)$. We will see that as $r \rightarrow \infty$, these hyperspheres converge to a limiting hypersurface, namely $M_{n}^{*}$. by

Earlier in this section, we discovered that the hypersurface of $H_{1}^{2 n+1}$ defined

$$
M_{0}(r)=\left\{\left.z \in H_{1}^{2 n+1}\left|\tanh ^{2}(r)\right| z_{0}\right|^{2}=\sum_{i=1}^{n}\left|z_{i}\right|^{2}\right\}
$$

is actually the lift (up to a congruence, of course) of a geodesic hypersphere of radius $r$. Notice that $\gamma(r) \in M_{0}(r)$ and that $\pi(P)$ is equidistant from every point on $M_{0}(r)$. So $\pi(P)$ plays the role of center of $\pi\left(M_{0}(r)\right)$ in $\mathbf{C} H^{n}$.

In particular, we see that the family of hypersurfaces $\left\{\pi\left(M_{0}(r)\right) \mid r>0\right\}$ is not our candidate for the convergent family. However, all is not lost, for we should be able to find a rigid movement of $\mathrm{CH}^{n}$, induced by an $A(r) \in U(1, n)$, that for each $r>0$ will translate $\pi\left(M_{0}(r)\right)$ to a geodesic hypersphere of radius $r$ and center $\gamma(r)$ in such a way that the family $\left\{\pi\left(A(r)\left[\left(M_{0}(r)\right)\right]\right) \mid r>0\right\}$ will converge to a limiting hypersurface. This limiting hypersurface will be $M_{n}^{*}=\pi^{-1}(N)$, which must then be a horosphere through $\pi(P)$.

For each $r>0$, let $A(r) \in U(1, n)$ be defined by

$$
A(r)=\operatorname{diag}\left\{\left[\begin{array}{ll}
\cosh (r) & -\sinh (r) \\
\sinh (r) & -\cosh (r)
\end{array}\right], I_{n-1}\right\} .
$$

$A(r)$ is a rigid motion that maps $M_{0}(r)$ onto the lift of the geodesic hypersphere of radius $r$ centered at $\gamma(r)$ and therefore induces a rigid motion of $\mathrm{CH}^{n}$ that moves the geodesic hypersphere $\pi\left(M_{0}(r)\right)$ that has radius $r$ and center $\pi(P)$ 


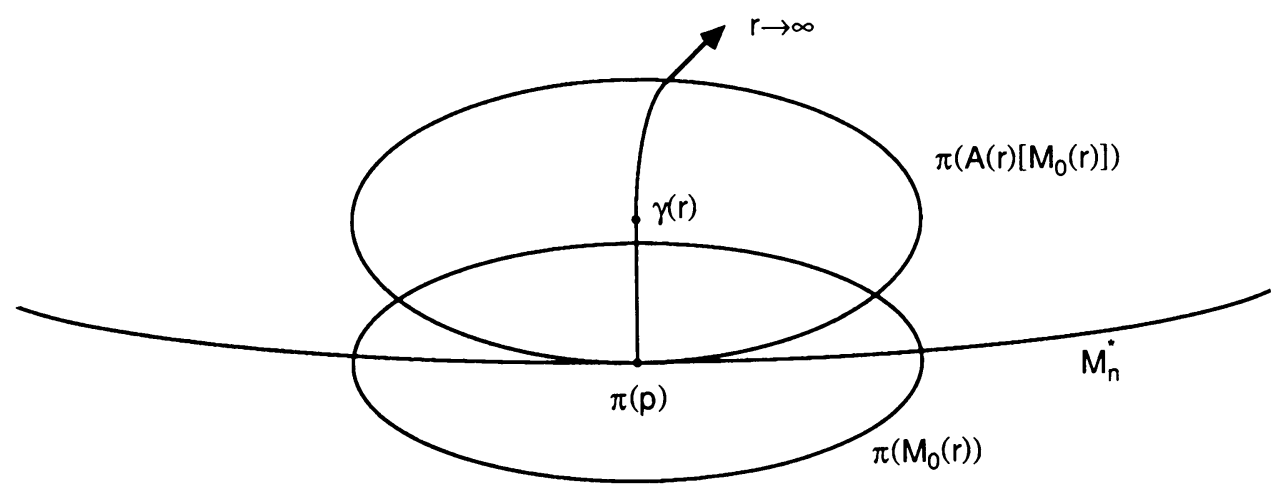

FIGURE 4

onto the geodesic hypersphere that has radius $r$ and center $\gamma(r)$ and contains $\pi(P)$.

Proposition. $N$ is the limiting hypersurface of the family $\left\{A(r)\left[M_{0}(r)\right] \mid r>0\right\}$ of hypersurfaces in $H_{1}^{2 n+1}$, that is,

$$
\lim _{r \rightarrow \infty}\left\{A(r)\left[M_{0}(r)\right]\right\}=N
$$

and is therefore an $S^{1}$-fiber bundle over a horosphere.

Proof. For any $z=\left(z_{0}, z_{1}, \ldots, z_{n}\right) \in M_{0}(r)$ we have

$$
\left|z_{0}\right|^{2}=\cosh ^{2}(r) \text { and } \sum_{j=1}^{n}\left|z_{j}\right|^{2}=\sinh ^{2}(r) \text {. }
$$

Let $w=\left(w_{0}, w_{1}, \ldots, w_{n}\right) \in A(r)\left[M_{0}(r)\right]$. Then

$$
w=\left(\cosh (r) z_{0}-\sinh (r) z_{1}, \sinh (r) z_{0}-\cosh (r) z_{1}, z_{2}, \ldots, z_{n}\right)
$$

for some $z \in M_{0}(r)$. In particular, we have

$$
\left|w_{0}-w_{1}\right|=(\cosh (r)-\sinh (r))\left|z_{0}+z_{1}\right|=e^{-r}\left|z_{0}+z_{1}\right| .
$$

Thus,

$$
\left|w_{0}-w_{1}\right| \leq e^{-r}\left(\left|z_{0}\right|+\left|z_{1}\right|\right) \leq e^{-r}(\cosh (r)+\sinh (r))=1,
$$

which shows that the limiting hypersurface $\lim _{r \rightarrow \infty}\left\{A(r)\left[M_{0}(r)\right]\right\}$ must satisfy $\left|z_{0}-z_{1}\right| \leq 1$ for any $z=\left(z_{0}, \ldots, z_{n}\right) \in \lim _{r \rightarrow \infty}\left\{A(r)\left[M_{0}(r)\right]\right\}$.

To see the reverse inequality, let $R>0$ be given. For each $r>R$, consider the disc

$$
S(r, R)=\left\{w \in A(r)\left[M_{0}(r)\right] \mid d(P, w)<R\right\}
$$

on the translated lift of the geodesic hypersphere $\pi\left(M_{0}(r)\right)$. Let $w \in S(r, R)$. Since $A(r)$ is an isometry, there is a $z \in M_{0}(r)$ such that $w=A(r) z$ and

$$
d((\cosh (r), \sinh (r), 0, \ldots, 0), z)<R .
$$


Hence, $\left|z_{0}-\cosh (r)\right|<R,\left|z_{1}-\sinh (r)\right|<R$, and $\left|z_{k}\right|<R$ for $k \geq 2$. Thus, for $r$ sufficiently large,

$$
\left|w_{0}-w_{1}\right|=e^{-r}\left|z_{0}+z_{1}\right| \geq e^{-r}(\cosh (r)+\sinh (r)-R)=1-e^{-r} R .
$$

Now we see that for a point $z$ in the lift of the horosphere within $R$ units of $P$, we must have $\left|z_{0}-z_{1}\right| \geq 1$. But $R$ was an arbitrary choice so that

$$
N=\bigcup_{R>0}\left\{\lim _{r \rightarrow \infty} S(r, R)\right\}=\lim _{r \rightarrow \infty}\left\{A(r)\left[M_{0}(r)\right]\right\} .
$$

This establishes the existence of a horosphere analytically in $\mathrm{CH}^{n}$.

As one would expect, the representation of a horosphere as a submersed level hypersurface depends both on the choice of $P \in H_{1}^{2 n+1}$ and on the geodesic emanating from $P$; equivalently: on the choice of normal to the lift of a horosphere at $P$. It is interesting to note that we obtain different bounds for $\left|z_{0}-z_{1}\right|^{2}$ for a limiting hypersurface of a convergent family of $S^{1}$-fiber bundles over geodesic hyperspheres if a different geodesic emanating from $P$ is selected.

\section{REFERENCES}

1. T. Cecil and P. Ryan, Focal sets and real hypersurfaces in complex projective space, Trans. Amer. Math. Soc. 269 (1982).

2. B. Y. Chen, G. Ludden, and S. Montiel, Real submanifolds of a Kaehler manifold, Algebras, Groups and Geometries 1 (1984), 176-212.

3. B. Y. Chen and K. Ogiue, Two theorems on Kaehler manifolds, Michigan Math. J. 21 (1974), 225-229.

4. B. Y. Chen and L. Vanhecke, Differential geometry of geodesic spheres, J. Reine Angew. Math. 325 (1981), 28-67.

5. J.-H. Eschenburg, Horospheres and the stable part of the geodesic flow, Math. Z. 153 (1977), 237-251.

6. R. Escobales, Riemannian submersions with totally geodesic fibers, J. Differential Geom. 10 (1975), 253-276.

7. A. Gray and L. Vanhecke, The volumes of tubes about curves in a Riemannian manifold, Proc. London Math. Soc. (3) 44 (1982), 215-243.

8. E. Heintze and H.-C. Im Hof, Geometry of horospheres, J. Differential Geom. 12 (1977), 481-491.

9. R. Howard, The Weingarten map of a tube, personal communication.

10. S. Kobayashi and K. Nomizu, Foundations of differential geometry, Vols. I and II, Wiley, New York, 1969.

11. W. Klingenberg, Riemannian geometry, De Gruyter Studies in Mathematics 1, De Gruyter, 1982.

12. Y. Maeda, On some real hypersurfaces of a complex projective space, J. Math. Soc. Japan 28 (1976).

13. S. Montiel and A. Romero, On some real hypersurfaces of complex hyperbolic space, Geom. Dedicata 20 (1986), 245-261.

14. K. Nomizu, Elie Cartan's work on isoparametric families of hypersurfaces, Proc. Sympos. Pure Math., vol. 27, Amer. Math. Soc., Providence, R.I., 1975.

15. B. O’Neill, Semi-Riemannian geometry, Academic Press, 1983.

16. __ The fundamental equations of a submersion, Michigan Math. J. 13 (1966), 459-469. 
17. W. Qi-Ming, Isoparametric hypersurfaces in complex projective spaces, Proc. 1980 Beijing Symposium on Differential Geometry and Differential Equations, Science Press, 1982.

18. L. Vanhecke and T. Willmore, Jacobi fields and geodesic spheres, Proc. Roy. Soc. Edinburgh Sect. A 82 (1979), 233-240.

Division of Natural Sciences, Lewis Clark State College, Lewiston, Idaho 83501 